\title{
RSSI-BASED WLAN INDOOR POSITIONING USED WITHIN A DIGITAL MUSEUM GUIDE
}

\author{
U. Grossmann ${ }^{1)}$, S. Gansemer ${ }^{1)}$, O. Suttorp ${ }^{2)}$ \\ ${ }^{1)}$ University of Applied Sciences and Arts Dortmund, Emil-Figge-Str. 44, 44227 Dortmund - Business Department, \\ \{uwe.grossmann, sebastian.gansemer\}@fh-dortmund.de \\ ${ }^{2)}$ University of Applied Sciences and Arts Dortmund, Emil-Figge-Str. 44, 44227 Dortmund - Computer Science \\ Department, oliver.suttorp@stud.fh-dortmund.de
}

\begin{abstract}
As modern mobile devices are often equipped with a WLAN interface and many buildings are equipped with a WLAN infrastructure, WLAN could be used for localization. The aim of this work is to investigate the quality of different indoor positioning methods based on values of WLAN received signal strength index (RSSI) using commercially available devices (smart phones, personal digital assistants). Three positioning algorithms were considered: minimal Euclidian distance, intersections of RSSI-Isolines and a stochastic model based on Bayes' theorem. A measuring test bed within an empty seminar room, four different types of personal digital assistants (Dell, Fujitsu, HP, T-Mobile) and two types of access points (Netgear, Lancom) were used. No modification of hardware and software was conducted. Furthermore this paper shows how WLAN localization can be applied in a museum guide application. This paper also shows the visualization of spatial user position frequency pattern during a field trial within a museum.
\end{abstract}

Keywords: RSSI, WLAN, Indoor, Positioning, Localization, PDA, Digital Museum Guide, Location Based Services, User Position, User Behavior

\section{INTRODUCTION}

For Location Based Services it is essential to know the current position of the user. Depending on the application the needed accuracy differs from less than one meter to some hundred meters. For outdoor applications satellite positioning systems or localization within cellular networks (e.g. cell of origin) can be used. Indoors satellite positioning systems do not work. Localization based on cellular networks requires a mobile device with an appropriate communication interface (GSM/UMTS) and an appropriate SIM card which leads to additional costs. For location based museum guide applications the current user position is needed as accurate as possible.

One appropriate localization system for indoor applications is WLAN localization. The aim of the activities presented in this paper was the investigation of different wireless local area network (WLAN) positioning methods for indoor applications using commercially available devices like personal digital assistants (PDA) without modifying their system hardware and software. The increase of existing WLAN infrastructures inside of buildings and the commercial availability of WLAN capable PDA lead to the question, whether indoor positioning using such infrastructures and devices would be accurate enough for mobile indoor applications.

Beside other methods some are based on recordings of values of received signal strength index (RSSI) of several access points. Several mathematical methods exist for calculating the current position. We investigated the accuracy of determining the current position using three different algorithms.

Several measurements were carried out over the period of a year's time. Circumstances, methods, results and conclusions are summarized below.

Having determined the current user position, this information can be logged into a database. Afterwards these user positions can be analysed. In this paper we present a visualization of spatial user position frequency pattern recorded during a field trial carried out in a museum.

\section{RELATED WORK}

Other groups working on the above mentioned questions use notebooks or laptops as mobile devices, and most of them use modified system hardware or software, e.g. a WLAN adapter with a modified driver. Some of them are outdoor oriented, 
e.g. for localizing a mobile phone.

A signature based outdoor location scheme is proposed by Meurer et al. which relies on covariance matrices of channel impulse responses (CIR) as signatures and which can do with a data base of substantially reduced size. This reduction resorts to the observation that the CIR are made up by individual wave fronts which remain unaltered over geographical areas with dimensions much larger than one wavelength. It is shown that the achievable localization accuracy in typical mobile radio scenarios satisfies the FCC E911 requirements ([1]).

Ladd et al. ([2]) used a notebook for determining the position by measuring the signal strength of several access points. For calculation they used Fingerprints and a Bayesian inference algorithm. Experiments were conducted by a human operator carrying a Hewlett Packard OmniBook 6000 laptop with a PCMCIA LinkSys wireless Ethernet card with a modified standard Linux kernel driver. This modification supports new functionalities, including the scanning and recording of hardware MAC addresses and signal strengths of packets and the automatic scanning of base stations. Sampling times ranged from ten seconds to about a minute. A total of 1307 packets over both orientations at 11 different positions were measured. Neighbouring positions had 3 metres distance from each other. The algorithm reported positions with a distance of up to 1.5 meters to the measuring position.

Retscher et al. ([3]) developed the IPOS system using RSSI-fingerprints as well. Their test bed was an office building and a tablet-pc was moved from each position to the next either in stop-and-go or kinematical mode. They focussed on determining whether a user is located inside a room or not and whether one or two calibration points within one room are sufficient.

Teuber et al. ([4]) used a method of minimal Euclidian distance together with Fuzzy logic postprocessing. Their test bed was an empty airport hangar. Accuracy of positioning was $4.47 \mathrm{~m}$ using Euclidian distance alone. With Fuzzy logic post processing the accuracy decreased to $3 \mathrm{~m}$.

The Ekahau Positioning Engine (EPE) is a commercially available Software for WLAN RSSI based indoor positioning. According to the manufacturer's instructions ([5]) the engine combines signal strength pattern recognition together with an attempt to include the user's history (boundary conditions like allowed paths and speed). Determination of the current position is possible depending on the environment with accuracy of 1-5 $\mathrm{m}$.

Similar measurements using similar methods as reported within this paper are performed by Roehrig et al. ([6]). They focused on a calibration grid with larger mesh widths and used a modified ISOLINEmethod (see below). The localization algorithms have also been discussed in [7].

Location sensitive museum guides with WLAN localization are currently very rarely used. One system is in use at museum "Tate Modern" in London. They evaluated their PDA-based museum guide in 2002 [8]. The main focus of their evaluation was the content use. They did not evaluate user behaviour or movements. Currently location sensitive museum guides are very rarely used; published evaluation results are rarely available. However there are some outdoor tour guides. Modsching et. al. [9] analysed the spatial behaviour of tourists in the city of Görlitz using GPS trackers and built a network of areas of interest. He also showed the positions on a density map. Larson, Bradlow and Fader [10] analysed the paths of shoppers in a supermarket using RFID tags attached to their shopping carts.

\section{METHODOLOGY}

There are different mathematical methods for positioning based on measuring RSSI values of several access points. We investigated the accuracy of determining the position under different circumstances, with different devices and mathematical methods.

One proposed evaluation method (fingerprint) is divided into two phases. During an initial calibration phase a radio map of RSSI values of several access points is developed at points with known coordinates. During the second phase (positioning phase) again the RSSI values of several access points are recorded. These observations are compared with those values stored previously during the calibration phase. The position whose calibration measurement is most similar to the measurement during positioning phase is assumed to be the current position.

One measure of similarity to be used is the EUCLIDIAN DISTANCE of observations.

$$
d=\sqrt{\left(S S_{c i 1}-S S_{m 1}\right)^{2}+\ldots+\left(S S_{\text {cin }}-S S_{m n}\right)^{2}} ;
$$

$S S_{c i j}$ represents an RSSI value of access point $j$ recorded during calibration phase at calibration point i. $S S_{m j}$ represents an RSSI value of access point $j$ recorded during positioning phase. There will be at least one calibration position $i$ with minimal $d$. One approach is claiming this point to be the estimated position.

Using the BAYESIAN THEOREM we generated a probability distribution over state space for measuring at point $(x, y, \theta)(\mathrm{x}, \mathrm{y}$ : coordinates of calibration point; $\theta$ : orientation of measurement) 
regarding one access point by scalar multiplication of RSSI histograms of the calibration and the positioning phase. Furthermore we assume that every position is equally likely with an a priori probability $\gamma_{i}$. The probability for the current measurement performed at point $(x, y, \theta)$ is calculated by:

$$
P\left(s_{i} \mid o_{j}\right)=\frac{\gamma_{i} P\left(o_{j} \mid s_{i}\right)}{\sum_{\alpha=1}^{n}\left(\gamma_{\alpha} P\left(o_{j} \mid s_{\alpha}\right)\right)} ;
$$

The product of these probabilities over all access points delivers the joint probability for measuring at point $(x, y, \theta)$. Points with maximum probability are assumed to be the current measuring position (see [2]).

For using the method of ISOLINES a network of triangles having calibration points as nodes is developed (Delaunay-Triangulation, see [11]). Given a recorded RSSI value of one access point we can select triangles whose nodes show signal strength values higher and lower than the recorded value. Linear interpolation between node values within the triangle delivers a detailed radio map consisting of a surface of interpolated RSSI values over the triangle. Moreover, we calculate an interpolated line of constant RSSI values (ISOLINE) within the triangle and the whole area of triangulation.

Given RSSI values of several access points triangles are selected including the corresponding isolines. Within triangles including several isolines the positions of intersection (see Fig. 1 below) are calculated ([12]).

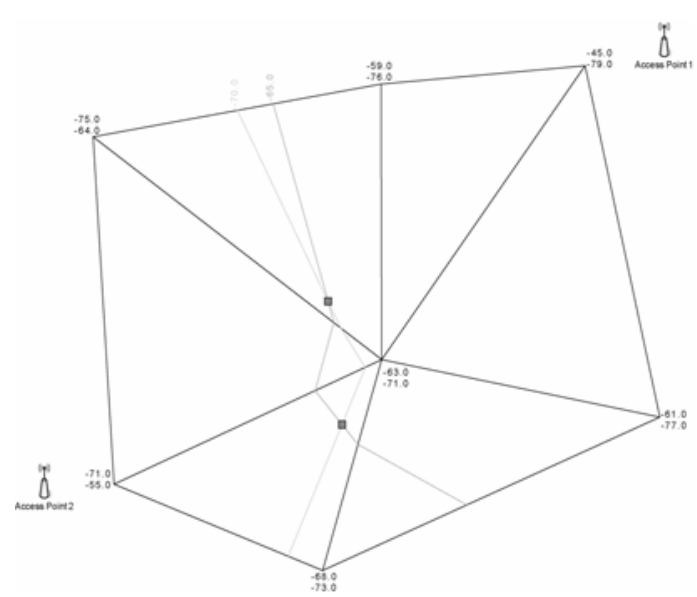

Fig. 1. - Triangles with isolines of two access points.

We select triangles including as much isolines of access points as possible. Within this list of selected triangles we establish a ranking based on the number of intersections of two isolines.

The balance point of the "best" triangle, i.e. the triangle with the largest number of intersection points, is considered to host the estimated position (see [13] and [14]).

\section{TEST BEDS AND MEASUREMENTS}

For applying and evaluating the algorithms discussed above we used two test beds. Measurements were carried out within an empty seminar room (scale: approx. $15 \mathrm{~m} \times 7 \mathrm{~m}$ ) within a building of Dortmund University of Applied Sciences, test bed 1.The location had been equipped with four access points at the outer corners below the room's ceiling. A map of the seminar room together with the calibration grid on the room's floor is shown in Fig. 2 below.

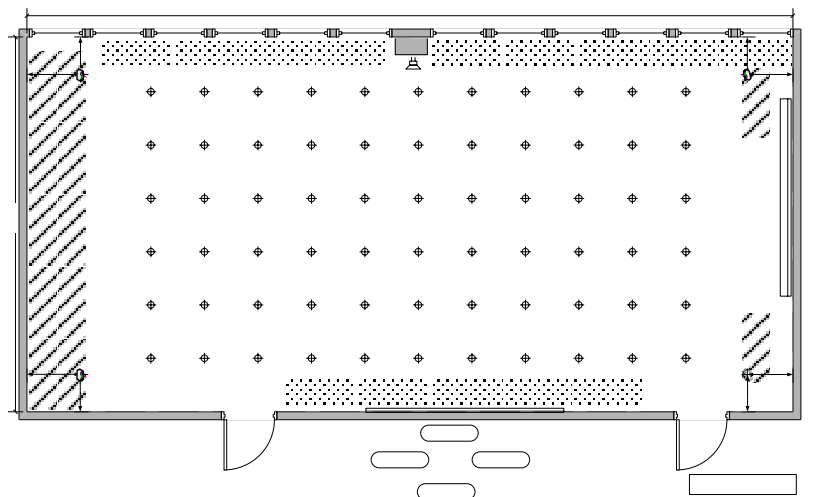

Fig. 2. - Empty seminar room (test bed 1).

Moreover an interpolated radio map of an access point below the room's ceiling at the lower left corner of the room is plotted. Areas colored black show high RSSI-values (approx. -40 db), areas colored grey or white show lower RSSI-values (approx. -60 db). The radio map is shown in Fig. 3.

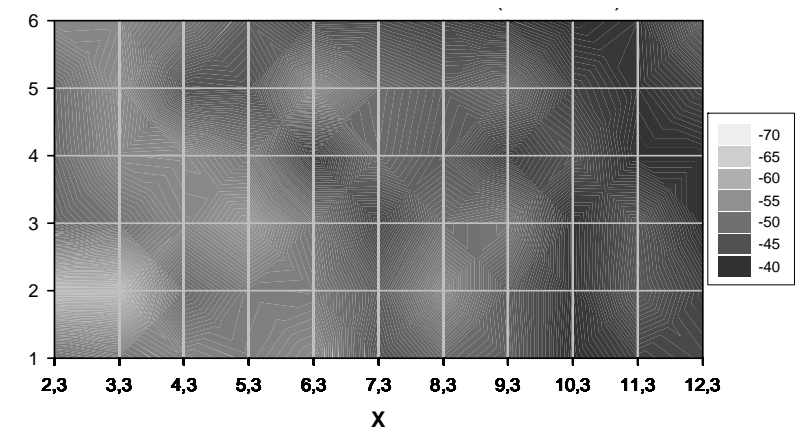

Fig. 3 - Interpolated radio map of test bed 1 for one access point (right upper corner).

Test bed 2 was a showroom (scale: approx. $30 \mathrm{~m}$ $x$ 11m) of the museum 'Strom und Leben' partly owned by the German energy supplier RWE: Again the location had been equipped with four access points at the outer corners below the room's ceiling. Fig. 4 shows a map of test bed 2. 


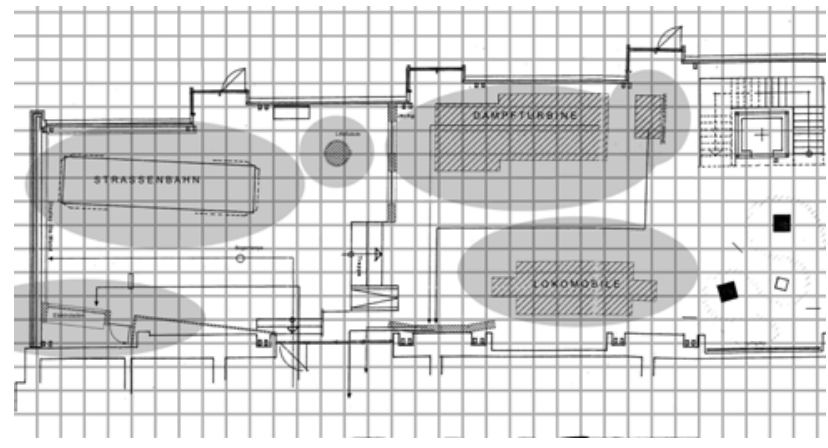

Fig. 4 - Museum's show room (test bed 2).

An interpolated radio map of an access point below the room's ceiling at the lower left corner of the room is plotted. Areas colored black show high RSSI-values (approx. $-40 \mathrm{db}$ ), areas colored grey or white show lower RSSI-values (approx. -60 db). The radio map is shown in Fig. 5.

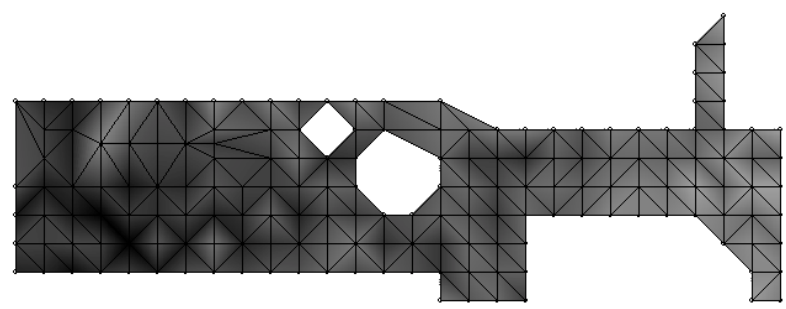

Fig. 5 - Interpolated radio map of test bed 2 for one access point (left lower corner).

In several series of measurements Netgear and Lancom access points were used.

For measuring WLAN signal strength values several Windows-Mobile PDA (Dell Axim X51v, HP 4700, Fujitsu/Siemens Pocket Loox 720, TMobile MDA 3) with inbuilt WLAN adapters were used. For measuring WLAN RSSI values of four access points a C\# software tool was developed using the OpenNetCF framework ([15]). The framework offers methods for recording data like SSID, MAC address and signal strength values of WLAN access points.

Throughout a pretest phase we checked the receiving qualities of the different PDA. With every device a radio map inside the lecture hall was measured and calculated. It turned out that the Dell PDA showed the most stable behavior during all measurements. It was able to record RSSI values decreasing reasonably with distance from the access point. All further measurements were carried out using this device.

The test environment was provided with a 1 metre rectangular grid on the floor. Measurements were carried out during calibration phase at each of these points in four orientations $\theta(0,90180,270$ degree). RSSI tuples of all 4 access points had been recorded for a period of 50 seconds (one reception per second). During positioning phase measurements were carried out again at a given position with given orientation. The recorded data were stored in a database. Table 1 shows typical fingerprints recorded throughout calibration phase.

Table 1. Fingerprints recorded in calibration phase

\begin{tabular}{|l|l|l|l|l|}
\hline Date/Time & MP & $\theta$ & MAC & $\mathrm{db}$ \\
\hline $\begin{array}{l}10.11 .2006 \\
14: 32: 13\end{array}$ & 001 & 0 & $\begin{array}{l}\text { 000B6B4D7A } \\
\text { D6 }\end{array}$ & -54 \\
\hline 10.11 .2006 & 001 & 0 & $\begin{array}{l}000 \mathrm{~B} 6 \mathrm{~B} 2 \mathrm{ADA} \\
\text { 14:32:13 }\end{array}$ & -45 \\
\hline $\begin{array}{l}\text { 10.11.2006 } \\
14: 32: 13\end{array}$ & 001 & 0 & $\begin{array}{l}000 \mathrm{~B} 6 \mathrm{~B} 2 \mathrm{ADD} \\
78\end{array}$ & -50 \\
\hline $\begin{array}{l}10.11 .2006 \\
14: 32: 13\end{array}$ & 001 & 0 & $\begin{array}{l}\text { 000B6B2ADA } \\
\text { 8B }\end{array}$ & -57 \\
\hline
\end{tabular}

\section{INDOOR MUSEUM GUIDE}

For evaluating the positioning technology presented above concerning usability and applicability we developed a digital museum guide application, which is based on a client server architecture. The clients are commercially available PDA with Windows Mobile operating system. Clients are designed as thin, i.e. they do not contain any data apart from a small software for recording RSSI data and for client/server communication. Thus, all content can be administrated centrally without the need for updating all clients if the content has changed.

The server runs a Windows Server operating system with Microsoft Internet Information Services. As database management system MySQL Server is used. In total there are four webservices running on the server. The webservices are for localization, content delivery, content management and analysis. The latter two are not necessary to operate the museum guide; they are only needed for analysis or administration. The webservices as well as the client software is programmed in $\mathrm{CH}$. The entire architecture is shown below in Fig. 6 .

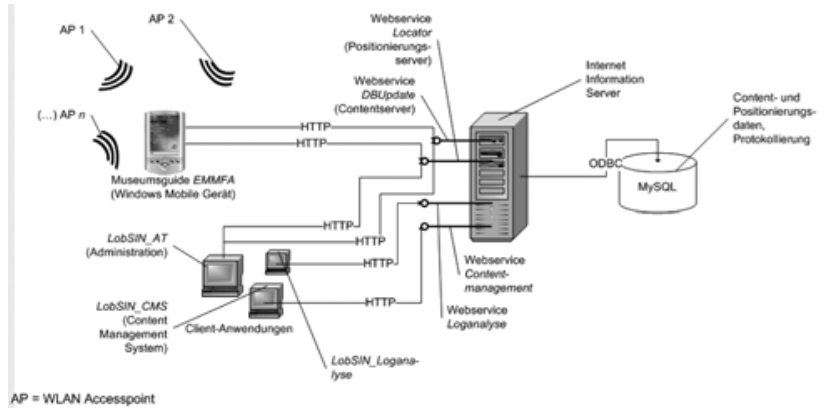

Fig. 6. - Client/Server Architecture of the museum guide.

The communication between client and server takes place via a IEEE $802.11 \mathrm{~b} / \mathrm{g}$ WLAN network.

The current user position is calculated every two seconds. Depending on the current position the PDA shows the objects in the surrounding area. Because of the limited accuracy of the positioning methods 
areas within the showroom (diameter: app. $5 \mathrm{~m}$ ) are located and the user has to select exhibits within these areas. The museum guide can present audio, video, images and texts.

\section{MUSEUM FIELD TRIAL}

A field trial was carried out within the museum (Umspannwerk Recklinghausen - Museum 'Strom und Leben' in Recklinghausen, Germany). Visitors were offered the PDA based museum guide when they walked in. In total 136 visitors participated in the field trial.

As in the field test before, four access points at the outer corners below the room ceilings were mounted. Additionally we equipped the museum with one access point in the middle of the exhibition space below the ceiling. This access point was used for data transmission between PDA and server. It proofed to be sufficient to cover the whole exhibition area with one access point. For localization, the Euclidian distance algorithm was used.

We evaluated user acceptance using a questionnaire. Moreover, all user actions such as clicks etc. were logged and stored in a database. Furthermore the calculated user position was logged. Based on these loggings user positions are visualized on a map. This may help to analyse user behaviour within the museum.

All calculated positions logged in the database are described by $\mathrm{x}$ and $\mathrm{y}$ coordinates referring to the lower left corner of the museum's map.

For visualizing the user position frequency pattern during the field trial we counted all calculated positions stored in the database within squares of $1.5 \mathrm{~m} \times 1.5 \mathrm{~m}$. The squares covered the entire field trial area and did not overlap each other. The central points of the squares build a grid of grid length $1.5 \mathrm{~m}$. Using Delaunay Triangulation we can find a set of triangles covering the area having the grid points as nodes. Similar to the visualization of the RSSI values by a radio map shown above we now are able to calculate a frequency map by 2dimensional linear interpolation between adjacent grid points.

Depending on the frequency gray values are allocated to specific areas according to a scale ranging from white for a frequency of zero to black for a frequency of more than 2000.

After overlaying this frequency map with a map of the museum we are able to identify areas within the museum which are frequently visited or others visited not at all (see Fig. 7 below).

\section{RESULTS}

The main result of the work is that RSSI based
WLAN indoor positioning is possible using commercially available devices, like personal digital assistants or smart phones equipped with WLAN adapters and access points, without any modification of system hardware and software.

For a test series of a random walk the positioning algorithms EUCLID, BAYES and ISOLINE showed a mean distance between measuring position and calculated position of max. 3 metres (see Table 2).

Table 2. Mean distance between measuring position and calculated position

\begin{tabular}{|l|l|l|l|}
\hline & test series & $\begin{array}{l}\text { Mean } \\
\text { distance } \\
(\mathrm{m})\end{array}$ & $\begin{array}{l}\text { standard } \\
\text { deviation } \\
(\mathrm{m})\end{array}$ \\
\hline BAYES & $\begin{array}{l}\text { Seminar (random } \\
\text { walk) }\end{array}$ & 2,91 & 1,56 \\
\hline EUCLID & $\begin{array}{l}\text { Seminar (random } \\
\text { walk) }\end{array}$ & 2,33 & 1,51 \\
\hline ISOLINE & $\begin{array}{l}\text { Seminar (random } \\
\text { walk) }\end{array}$ & 2,00 & 1,04 \\
\hline
\end{tabular}

The calculated mean distances for the three algorithms differ by max. $1 \mathrm{~m}$. Nevertheless, we observe considerable standard deviations for the mean distances in all cases between 1 and $1.5 \mathrm{~m}$. A more detailed analysis including different test series within both test beds is shown in refs. [13, 14].

Fig. 7 shows the frequency map of calculated user positions during the field trial. Black colored areas had been frequently visited; white colored areas are not visited at all. On the left hand side of Fig. 7 there is the entrance hall with the reception desk and the cafeteria area, in the middle we have an area with interactive games and other features, and on the right hand side an old tram is located. These areas show a deep black color, i.e. these are the areas mainly visited.

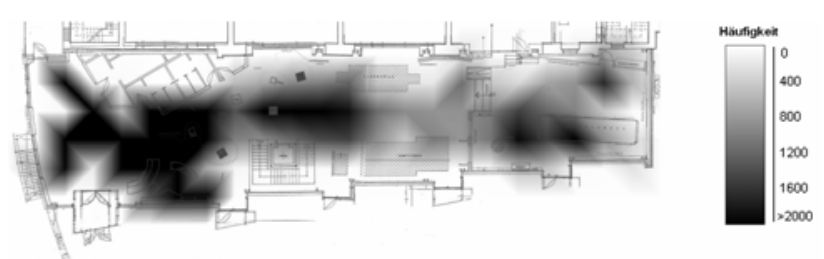

Fig. 7. - Visualization of user position frequency pattern.

Fig. 7 includes all data recorded during the field trial. This means that the figure represents the calculated positions of all 136 visitors.

A similar analysis could be performed using the data of only one particular visitor or a particular visitor group (e.g. only female/male persons). Combined with logged data concerning clicks on specific content items this may lead to a specific profile of interests which could be used for direct marketing measures or for a spatial redesign of the exhibition. 


\section{CONCLUSION}

The use of access points of different quality (Netgear, Lancom) led to unexpected results. Using more expensive and more capable access points (Lancom) in comparison to more simple and less expensive access points (Netgear) did not show a significant improvement of accuracy.

Measurements with personal digital assistants of different manufacturers showed different quality in receiving signal strength index values. Probably, for a PDA the antenna design has a stronger influence on the received signal quality than for notebooks.

Some series of measurements over time showed an unusual behaviour. Received signal strength index values did not change over a period of several seconds. Usually, a change within a range of 3-5 db every second was observed. There is some evidence, that the used OpenNetCF framework's methods are not mirroring instantly, i.e. within a period of a second or less, RSSI value changes. The framework OpenNETCF has to be examined again.

The localization algorithms can be used for indoor LBS applications. The calculated position can be used to present context related content to the user. The position can also be logged to a database for analyzing user behavior and user positions. Even if the localization is not used for presenting context related content the analysis of user positions may be of high value.

\section{ACKNOWLEDGMENT}

This work was funded by the German Federal Ministry of Education and Research (BMBF-Project IKAROS) and the commission for research, development and transfer of Dortmund University of Applied Sciences.

The Authors are grateful to Mr. Hanswalter Dobbelmann, head of "Umspannwerk Recklinghausen - Museum Strom und Leben” Recklinghausen, for his cooperation and support.

\section{REFERENCES}

[1] Meurer M., Heilmann S., Reddy D., Weber T., Baier P.W. A signature based localization technique relying on covariance matrices. in Kamakya K, Jobmann K., Kuchenbecker, H.-P. (eds). Joint 2nd Workshop on Positioning, Navigation and Communication (WPNC'05), Hannover, 17.3.05, p 31-40.

[2] Ladd A. M., K. E. Bekris, A. Rudys, L. E. Kavraki, D. S. Wallach. Robotics-based location sensing using wireless Ethernet. Wireless Networks, vol 11, no 1-2, 2005, p. 189- 204.

[3] Retscher G., Moser E., Vredeveld D. and
Heberling D. Performance and accuracy test of the WLAN indoor positioning system ipos. in Kamakya K, Jobmann K., Kuchenbecker, H.-P. (eds). Proceedings of the 3rd Workshop on Positioning, Navigation and Communication (WPNC'06), Hannover. 16.3.06, p 7-15.

[4] Teuber A., Eissfeller B. WLAN indoor positioning based on Euclidean distances and fuzzy logic. in Kamakya K, Jobmann K., Kuchenbecker, H.-P. (eds). 3rd Workshop on Positioning, Navigation and Communication (WPNC'06), Hannover, 16.3.06, p 159-168.

[5] Ekahau; "Ekahau positioning engine 2.0; 802.11 based wireless LAN positioning system"; An Ekahau Technology Document, 2002.

[6] Roehrig C., Kuenemund F. Mobile robot localization using WLAN signal strength. Personal Communication, FH Dortmund 2006, unpublished.

[7] Grossmann U, Schauch M. RSSI based WLAN indoor positioning with personal digital assistants, in: Proceedings of the $4^{\text {th }}$ IEEE Workshop on Intelligent Data Acquisition and Advanced Computing Systems: Technology and Applications IDAACS 2007, (Ed. Sachenko, A), pp. 653 - 656; Dortmund, 2007.

[8] Tate Modern: Tate Modern Multimedia Tour Pilots 2002-2003, Online: http://www.tate.org. uk/modern/multimediatour/phase1_keyfindings .pdf, last reviewed 15.04.2008.

[9] Modsching M., Kramer R. ten Hagen K. Gretzel U. Using geographic tracking data to analyse spatial behaviour in eTourism, Online: http://www.ikaros-projekt.de/CmodsDownload -index-req-getit-lid-62.html, last reviewed 15.04.2008.

[10] Larson S., Bradlow E.T., Fader P.S. An exploratory look at supermarket shopping paths. in: International Journal of Research in Marketing, Vol. 22, Issue 4, December 2005, pp.395-414.

[11] Klein R. Algorithmische Geometrie, AddisonWesley, Bonn, 1997.

[12] Dalhaus M. WLAN-Ortung innerhalb von Gebäuden mittels Signalstärkelinien. Internal Report, FH Dortmund 2006, unpublished.

[13] Grossmann U., Roehrig C., Hakobyan S., Domin T., Dalhaus M. WLAN indoor positioning based on Euclidian distance and interpolation (isobars). In Wollert J. (ed). Wireless Technologies, Dortmund, 27./28.9.06, Fortschritt-Berichte VDI, Reihe 10, Nr. 772, S.296-305, 2006.

[14] Grossmann U., Schauch M., Hakobyan S. The accuracy of algorithms for WLAN indoor positioning and the standardization of signal 
reception for different mobile devices. International Journal of Computing, Vol . 6 (2007), Issue 1, pp 103-109.

[15] L'hoest A. Entwicklung mobiler Anwendungen mit Microsoft.NET. Internal Report, $\mathrm{FH}$ Dortmund 2005, unpublished.



Prof. Dr. Uwe Grossmann, studies of mathematics and information technology (Bochum University, Germany), PhD in mathematics (Bochum University, Germany), research associate at Essen University and Max-Planck-Institute Dortmund, project manager computer aided manufacturing at mbp software \& systems gmbh.

Current position: professor of mathematics and business information technology at University of Applied Sciences and Arts, Dortmund; project manager of joint BMBF research project IKAROS; speaker of research group 'mobile business - mobile systems' of University of Applied Sciences and Arts, Dortmund.

Focus of research: multimedia applications, mobile business/commerce, mobile systems. author of a textbook in information technology and several papers in the area of mobile/wireless systems; reviewer and session chair of national/international conferences

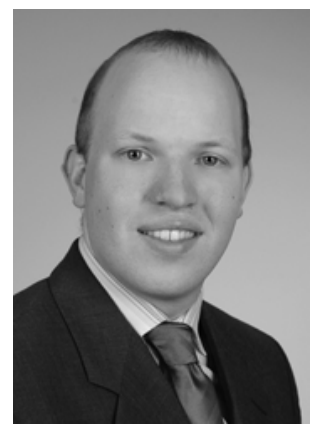

Dipl. Bw. (FH) Sebastian Gansemer studies of business administration with focus on business information technology at University of Applied Sciences and Arts, Dortmund.

Current position: research associate at Business Department of University of Applied Sciences and Arts, Dortmund.

Focus of research: mobile business, mobile commerce, location based services.

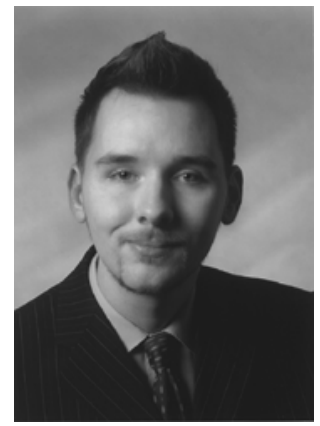

Cand. inf. (FH) Oliver Suttorp graduate in computer science at University of Applied Sciences and Arts, Dortmund. 